\title{
LAS NOCIONES DE LOS NIÑOS \\ ACERCA DE LO VIVO. \\ IMPLICACIONES DIDÁCTICAS
}

\author{
Norma Constanza Castaño Cuellar y Mireya Leudo Arenas ${ }^{*}$
}

\begin{abstract}
The article takes some of the results from the research "Biological thinking and communicative development in children". The results indicate that children have a coherent thinking about the notion alive, eventhough it is obviously different from the scientific concept. This coherence is demonstrated when children try to define the notion of being alive in doing so, they use the most evident traits such as movement. Besides, their thinking gradually pays attention to other characteristics, equally evident, such as growth, feeding needs, reproduction and death.

The paper states that in spite of the fact that they have the same age, they are in the same educational institution and they are in the same grade, this children do not have a unique nor simple criteria, to define the notion of alive. Their thinking is not simple; it has certain complexity and shows that they have their own vision of the meaning of being alive. This vision is different from the adult's vision and, obviously quite different from the scientific concept academically accepted. Nevertheless, these children are actually structuring ideas about the holistic nature of living beings and their relations with the environment.
\end{abstract}

\section{PALABRAS CLAVES}

Ciencia de lo vivo, pensamiento del niño, categorías, animistas, utilitaristas.

\section{ANTECEDENTES}

La investigación se hace posible cuando el Instituto Pedagógico Nacional se interesa por involucrar en la educación de los niños la formación de actitudes hacia las ciencias a través de un espacio denominado "Quike, el Científico" proyecto iniciado por el Departamento de Pre-escolar de la Universidad Pedagógica Nacional en 1992.

La investigación se propone reorientar el papel del conocimiento biológico ${ }^{1}$ en la formación de las nuevas generaciones, reorientar los procesos de construcción del

\footnotetext{
* Docentes del Departamento de Biología de la Universidad Pedagógica Nacional Grupo de Investigación Enseñanza de la Biología en Colombia

Digitalizado por RED ACADEMICA
} 
conocimiento para hacer posible por ejemplo, explicar la cotidianidad y por qué no, lograr que el niño empiece a desarrollar proyectos de vida conscientes y autodeterminados, coherentes con el contexto sociocultural.

Actualmente los practicantes de biología realizan allí proyectos relacionados con las nociones, y atención que no decimos preconceptos ${ }^{2}$, de los niños acerca de las plantas, al igual que propuestas metodológicas para la formación de actitudes científicas a través de la lectura y la escritura, entre otros. Al mismo tiempo el proyecto pasa a denominarse "Club de Quike" por la idea de participación a que el término da lugar.

\section{EL PROBLEMA DE INVESTIGACION}

Sabemos que la escuela selecciona los lenguajes y modos de expresión del niño y los centra principalmente en la lectura, escritura y las matemáticas ${ }^{3}$. A las expresiones más auténticas del niño, se les presta poca atención y así la enseñanza básica desde el inicio, no le posibilita al niño el imaginar y el crear. El afán de esta escuela moderna ${ }^{4}$ es "culturizar", mostrarle al niño que existe un mundo al que él difícilmente puede acceder, porque es un mundo que no se relaciona para nada con su cotidianidad; y que deja de lado sus genuinos intereses; así por ejemplo para el caso que nos atañe, hemos demostrado que estas son inicialmente inquietudes acerca de los fenómenos naturales y especialmente sobre lo vivo. El maestro se convierte así en el portador de la cultura vigente, cultura ajena al mundo del niño, siempre lista a dar respuestas que el niño debe repetir, lo que no le posibilita utilizar su imaginación e intentar la búsqueda de soluciones a sus propias inquietudes.

En la clase de ciencias que se imparte en los primeros niveles de educación, es notorio que la didáctica también se centra en la transmisión de contenidos y el aprendizaje en el rendimiento y que los intereses espontáneos del niño que giran alrededor de lo vivo y de los fenómenos de la naturaleza no se tienen en cuenta. Tampoco se ha fijado la atención en las formas como el niño construye pensamiento acerca de lo vivo; aspecto importante en su formación, dado que es en los primeros años de vida cuando, por medio de los procesos de socialización, él empieza a relacionarse con el mundo viviente.

\footnotetext{
${ }^{1}$ Hacemos referencia a la enseñanza de la biología pensando que aunque el área obligatoria es la de ciencias naturales, de una u otra manera, el reconocimiento y la comprensión de lo vivo es fundamental en los primeros años de vida y deberla tener cabida en una estructura curricular

2 En este caso hablamos de nociones aludiendo al pensamiento genuino del niño; hablar de preconceptos significaría que el pensa miento del niño al no coincidir con los conceptos científicos es erróneo. Lo que queremos relevar es que el niño tiene un pensamiento propio producto de sus vivencias y que como tal debe ser valorado.

${ }^{3}$ Entendidos éstos como contenidos exclusivamente, sin preocuparse por el significado y por el valor que tienen en los actos de comunicación.

${ }^{4}$ La escuela en suma exige no sólo positivismo científico, sino rigor en el pensamiento, eficacia de resultados cuantificables en todo cuanto se hace; tratando seguramente de hacer realidad la fórmula mágica del progreso moderno De esta manera nos encontramos ahora si. con una oposición entre lo que somos y lo que la escuela, basada en modelos externalistas, nos exige..

La escuela en su ceguera reduccionista y objetivante perdió la capacidad de interpretación de los deseos de los sujetos y de las sociedades; perdió la capacidad de vislumbrar igualmente las necesidades de desarrollo económico y social. 
De otro lado es constante que por tratar de acercar al niño al conocimiento biológico, desconociendo el desarrollo cognitivo, se termina reforzando el antropocentrismo ${ }^{5}$ y dando una visión errada de lo vivo, negándosele así el acceso a una imagen científica de la realidad.

\section{LA NECESARIA FUNDAMENTACION}

\subsection{El pensamiento acerca de lo vivo}

Las concepciones acerca de lo vivo han variado a lo largo de la historia de la humanidad entre el animismo, el antropocentrismo, el vitalismo y el materialismo. Para C.U.M. Smith (1975), se han dado por lo menos cuatro biologías diferentes pero con sentido, la aristotélica, la cartesiana, la goetheana y la actual.

La biología aristotélica afirma, es profundamente teleológica la naturaleza no hace nada en vano' parece ser su fundamento. Para Descartes la imagen de lo vivo es el autómata cartesiano; para Goethe lo vivo se constituía en una búsqueda de formas arquetípicas. Hoy lo vivo se concibe en términos moleculares y evolutivos.

Evidentemente el conocimiento cotidiano acerca de lo vivo ha recorrido caminos distintos. Nos encontramos entonces con una conciencia mágica que no marca límites entre lo subjetivo y lo objetivo, entre el yo y el no yo. El mundo era considerado en actividad dinámica y teleológica, lo mismo que un organismo vivo; además el ser humano se consideraba inmerso en la naturaleza, conectado a ella a por hilos invisibles (Smith, 1975).

Es curioso como para Aristóteles, la naturaleza hace referencia a aquellas cosas que "poseen en sí mismas, ...una fuente de movimiento", aludiendo a una connotación tanto física como psicológica. Igualmente para Tales de Mileto las piedras imantadas poseen vida, o alma, porque son capaces de mover el hierro" (Smith, 1975, p. 60). Los objetos vivos se caracterizan entonces por la capacidad de Iniciar movimiento Al parecer afirma Smith estos dos aspectos se hallaban unidnos en los primeros tiempos lo psicofísico constituyendo el principio animador el aliento vital ${ }^{6 .}$

La historia de las ciencias como lo afirma Wuketits (1995), muestra que tanto biólogos como filósofos de la Biología defienden teorías particulares cuando definen e! concepto vida Las más difundidas se relacionan con el vitalismo y el mecanicismo Los vitalistas sostienen que la vida y tonas sus expresiones particulares desarrollo comportamiento, entre otras dependen de agentes específicos que no pueden ser explicados en términos físicos y químicos Estos agentes han tenido diferentes denominaciones a o largo de la historia: entelequia, pneuma, vis piastica, principio vital... La teoría mecanicista por su parte, se caracteriza por plantear que todo sistema viviente es un sistema mecánico, una clase de máquina basada en elementos químicos y que sigue las leyes universales de la

\footnotetext{
${ }^{5}$ Se refuerza el antropocentrismo cuando al tratar de facilitar la comprensión se acude a comparaciones con el modelo humano: es decir se pinta el sol con rostro humano e incluso anteojos para el sol por ejemplo

${ }^{6}$ Es interesante la anotación del autor respecto a que no encuentra en este período la necesidad de hacer una diferenciación entre animado e inanimado "para ellos (el mundo) poseía una actividad dinámica y teleológica, lo mismo que un organismo vivo".
} 
materia. De ahí que no consideran diferencias notables entre los objetos vivos y no vivos puesto que los organismos son reducibles a compuestos moleculares.

En esta controversia subyace para Wuketits(1995), que respecto al pensamiento cognitivo hay dos tipos de biólogos. Aquellos que se inclinan al análisis y al reduccionismo y aquellos que por tener una intuición hacia la consideración del todo tienden a sintetizar más que analizar.

\subsection{El pensamiento del niño}

Piaget (1933) en un estudio pionero encuentra que la noción de vida es familiar para los niños y determina que esta concepción se elabora a lo largo de cuatro etapas. En la etapa inicial dice, los niños entre los 6-7 años consideran como vivo todo lo que tiene actividad, función o utilidad definida en términos antropocéntricos. Encuentra un paralelismo entre la noción de vida y la noción de conciencia, y ve en esas razones una afirmación de la finalidad profunda de la naturaleza y de un continuo de fuerzas inmanentes a los objetos destinadas a realizar un fin Plantea que hay todavía indiferenciación entre la causalidad física y la conexión psicológica e intencional" (Piaget. 1993 p. 174).

En la etapa subsiguiente (niños entre los 6-8 años) que considera como una etapa en transición, encuentra que la vida se define por el movimiento. Considera que el movimiento contiene para el niño los caracteres de la vida, que el problema de la definición de vida les preocupa realmente y la asimilación de la vida al movimiento busca una significación; lo cual cree confirmar cuando en la tercera etapa los niños diferencian tipos de movimiento.

En la tercera etapa (8-9 años) al diferenciar entre el movimiento propio y el espontáneo, la vida se identifica con el primero. Finalmente, plantea que en la última etapa (11-12 años) la vida se reserva a los animales en primera instancia, o a los animales y plantas. Concluye diciendo que la noción de vida es intermediaria entre la causalidad mágica y el dinamismo de la fuerza sustancial.

Otros trabajos como el de Keil (Citado por Rodrigo, M.J. 1993) señalan que la distinción entre vivo (animal, planta) -no vivo no implica que los niños sean conscientes de la existencia de una categoría de lo vivo, porque argumenta las propiedades comunes son lo suficientemente abstractas para no ser percibidas directamente. Este planteamiento es discutible porque obviamente desde una óptica científica, los elementos comunes a la categoría son abstractos, pero desde una óptica cotidiana no solo se ubican en el terreno de lo real, sino que se hallan una correspondencia antropocéntrica, como lo muestran los resultados obtenidos en nuestra investigación.

Una de las investigaciones más importantes respecto al cambio conceptual en la infancia efectuada por Carey (1978) plantea que el origen de las respuestas animistas de los niños para definir lo vivo es el incompleto conocimiento biológico que poseen; lo trata de demostrar encontrando que aproximadamente a los 10 años ese pensamiento animista entra en declive. A este planteamiento contraponemos un cuestionamiento ¿por qué los adultos escolarizados aún tienen pensamiento antropomórfico a pesar de la escolarización creciente? 
Carey argumenta igualmente que la atribución de vida a objetos inanimados puede ser más un problema semántico que conceptual. Pensamos que seria importante abordar un estudio que lo demuestre, porque seguramente cuando se hace alusión a la palabra vivo, ésta no tiene un significado único. No encuentra Carey consistencia en los resultados de investigaciones similares a las de Piaget, como la de Laurendeau y Pinard. Más nos parece que esta conclusión está dada, en parte, por el enfoque cuantitativo utilizado en este tipo de estudios que tratan de ubicar el pensamiento de los niños en categorías homogéneas determinadas mediante análisis estadísticos.

\subsection{Consideraciones acerca del conocer}

La investigación parte del planteamiento de que no puede darse un conocimiento directo de los fenómenos Conocer no es ni reproducir ni representar, conocer es construir, pues toda referencia al mundo implica mínimamente un acto de clasificación y de ordenación (Melich. 1996). En este sentido todo lo real para ser calificado como tal debe ser conocido de algún modo; es decir debe encontrarse en relación con nosotros. Como plantea Schutz. (Citado en: Melich, 1990, p. 21) "lo que constituye la realidad es el sentido de nuestras experiencias, y no la estructura ontológica de los objetos; el objeto por el objeto...

Es así como el enfoque del realismo experiencial por ejemplo (Kleiber, 1995), plantea que las estructuras conceptuales provienen de nuestra experiencia y ubica como problema central del pensamiento el de la categorización. Considera que ésta es una operación mental compleja esencial y afirma, que sin la capacidad para superar las entidades individuales, concretas o abstractas, el entorno percibido seria caótico y permanentemente nuevo. De ahí se sigue que la biología no existiría como ciencia, de no ser por la categorización de la inmensa diversidad de lo vivo que hizo la historia natural y no seria muy aventurado suponer que para desarrollar pensamiento en torno a lo vivo es indispensable que desde la didáctica se fije la atención en las formas de categorización de lo vivo, desde los primeros niveles de escolaridad, también como forma de desarrollar estructuras cognitivas.

Estas concepciones nos llevan a plantear que el cuestionamiento que debe hacerse a la didáctica es ¿en qué forma el conocimiento biológico puede contribuir a la construcción del mundo de los niños? Y no la clásica pregunta ¿Qué deben aprender los niños de biología? Por lo tanto, la enseñanza para el nivel educativo primario estaría centrada en involucrar emocionalmente al niño con la realidad del entorno que lo rodea y aplicar el saber de las ciencias naturales en su vivencia diaria como conocimiento necesario por ejemplo, para reconocerse a si mismo como ser vivo y haciendo parte del entorno natural y social; caracterizar el entorno natural; mejorar su vivencia cotidiana.

\section{METODOLOGIA}

La investigación es una microetnografía que explora e interpreta el pensamiento de los niños acerca de lo vivo buscando rescatar las expresiones espontáneas de los niños.

Las nociones acerca de lo vivo se sistematizaron en categorías semánticas que respondían a las preguntas ¿Este objeto es vivo? ¿Por qué? efectuadas a los niños de cinco años y de siete años de edad. A los chicos de ocho años se les pidió que hicieran 
una lista de seres vivos y otra lista de seres no vivos, dos años después de haber empezado las prácticas pedagógicas con ellos.

Las prácticas pedagógicas ofrecieron alternativas para descentrar el pensamiento de los niños. Esencialmente se centraron en identificar los intereses que los niños tienen acerca de la naturaleza y proponer alternativas didácticas, acordes con sus inquietudes y con su pensamiento en un espacio donde los niños se expresan libremente y en donde se les orienta la búsqueda de respuestas a sus preguntas particulares. La didáctica en general se enfoca a la identificación, observación y descripción de los objetos vivos.

\section{RESULTADOS}

\subsection{La noción de vivo de los niños de cinco y siete años de edad}

Para los niños de cinco años y medio en general lo vivo son objetos que se mueven y en este sentido las plantas en general, están excluidas como tales. Es así como afirman por ejemplo, el pino "está vivo si se mueven las hojas' pero el cactus "no está vivo porque no se mueve". Algunos pocos empiezan a diferenciar el movimiento autónomo como característica esencial de lo vivo, dicen el carro no está vivo, "anda por una batería", el pez está vivo porque "nadie lo maneja, el solo nada".

Utilizan términos con alguna connotación biológica, el pino está vivo porque "crece si le echo agua" o el pez también lo está porque vive en el agua y puede respirar allí.

Es notorio el uso de rasgos antropomórficos para afirmar o negar el carácter vivo. Se encontraron afirmaciones del tipo, el sol no está vivo "no tiene boca ni ojos" el niño y el pez están vivos porque "tienen ojos", pero el cactus no lo es porque "no tiene piernas".

Se destaca la diversidad de categorías con que estos niños distinguen lo vivo. Se encontraron concepciones utilitaristas (el sol está vivo porque calienta a la gente), creacionistas (Dios maneja a la lluvia también, cuando hace viento apaga el sol y llueve), animistas (el pino no está vivo porque no le duele), de identificación de artefactos (el carro no está vivo porque es un aparato), requerimiento de cuidado, de energía (el niño está vivo porque saca energía de los alimentos), racionalidad (el niño está vivo porque piensa), entre otros.

Los niños de siete años en su mayoría, consideran vivos a los animales y no vivas a las plantas. Relacionan hojas, plantas y árboles con "palos" y son consecuentes cuando afirman que no están vivos. Las categorías encontradas aquí son muy similares a las de los niños de cinco años y medio de edad.

\subsection{La noción de lo vivo de los niños de ocho años}

Los niños de ocho años en su mayoría, identifican como vivos a animales y plantas, algunos pocos solo consideran como vivos a los animales. Hay coherencia entre la designación de lo vivo como activo y la designación de objetos inanimados en movimiento. Lo que nos muestra que la condición de movimiento no es ahora lo esencial. Las razones giran alrededor de categorías biologicistas que aluden a características morfológicas o a características esenciales de lo vivo como nacer y crecer, animistas 
(cuando las tocan les duele), antropocéntricas (no caminan ni oyen. no tienen cuerpo). utilitaristas (sirven a la naturaleza, nos dan vida)

En general no se encontraron referencias explicitas de tipo antonímico vivo-muerto. Las nociones de lo vivo aún cuando han desplazado el animismo inicial son predominantemente antropocéntricas, sin embargo hay una característica interesante, lo humano sirve ahora como punto de referencia, de comparación. Es decir, hay una diferencia clave, la visión antropocéntrica inicial asegura que la planta es viva porque "tiene brazos" por ejemplo; la segunda concepción intenta hacer una comparación, "nace como los humanos", "es como alguien que crece hasta ser muy viejo y de ahí uno se muere" Se pasa de una concepción sincrética inicial a tomar el referente humano como elemento de comprensión ".. es como".

Se demuestra igualmente que la práctica pedagógica logró desarrollar acciones didácticas que les permitieron a os niños diferenciar lo vivo de lo no vivo y lo que es más importante descentrar el animismo y el antropomorfismo inicial en un lapso de dos años.

\section{IMPLICACIONES}

Estos resultados nos llevan a plantear que los niños tienen un pensamiento coherente acerca de lo vivo, aunque obviamente diferente al concepto biológico. Esta coherencia se manifiesta en que para definir lo vivo, los niños acuden a lo evidente que inicialmente es el movimiento y en que paulatinamente su pensamiento va dirigiendo la atención a otras características, igualmente evidentes e inmediatas, como el crecimiento, la necesidad de alimentarse, el tener hijos o el morir.

Se muestra que los niños no tienen criterios únicos, ni simples para definir lo vivo, a pesar de que estos tienen la misma edad, están en la misma institución educativa y en el mismo grado de escolaridad Su pensamiento no es simple, tiene cierto grado de complejidad y muestra una visión del mundo viviente propia, distinta a la de los adultos y obviamente diferente al conocimiento biológico académicamente constituido. Aunque muestra algunas referencias que constituyen indicios acerca de la integralidad de lo vivo y de relaciones con el entorno que ocupan.

En esta investigación se han originado alternativas de las que va emergiendo la imagen de un niño con pensamiento propio, capaz de inventar y escribir historias iladas y coherentes que reflejan su mundo interior, pero que a la vez consideran los símbolos y signos con que la cultura empieza a influirlos, niños que piensan lo vivo con tintes muy particulares y hasta con filosofía, niños que sorprenden con su sabiduría ${ }^{7}$, en fin niños con un pensamiento distinto al que la escuela pretende imponer.

Lo anterior significa que el niño no se imita a recibir pasivamente estas influencias, es un sujeto activo, que reacciona, influye e interacciona con su entorno. La escuela debe reconocer que los niños tienen pensamientos propios, actitudes e intereses diferentes a los de sus maestros adultos; la escuela debe aportarle al niño nuevas experiencias y

\footnotetext{
${ }^{7}$ En tercero de primaria, por ejemplo, algunos niños afirman diferenciarse de los animales porque "piensan" o porque "sueñan".
} 
horizontes de vida que le posibiliten formarse como persona singular y en relación con los otros y con el entorno natural y cultural.

Es necesario comprender igualmente, que sólo en la interacción con los otros puede lograrse el aprendizaje, puesto que las estructuras cognitivas no están preformadas en el niño; él las construye a través de su acción sobre los objetos y de la coordinación de sus puntos de vista con los de los demás. En lugar de dejar que su memoria domine a la razón o de someter su inteligencia a unos ejercicios impuestos desde el exterior, es necesario que aprenda a hacer funcionar su razón por si mismo y a construir libremente sus argumentos.

Finalmente planteamos que seria necesario empezar a pensar en diseñar un currículo alrededor del pensamiento que los niños tienen acerca de lo vivo y de sus propios intereses acerca del mundo viviente, en un espacio de actividades libres donde se respete la diferencia, la espontaneidad, la libre expresión, como formas necesarias de comunicación.

\section{BIBLIOGRAFÍA}

CAREY, S. 1985. Conceptual change in childhood. MIT Press: Cambridge.

CASTANO, NC. La enseñanza de la Biología en Colombia. I Encuentro Distrital de Experiencias en Enseñanza de la Biología. Santafé de Bogotá, Septiembre 21 de 1995.

. Los niños y su pensamiento acerca de lo vivo. Implicaciones para la enseñanza primaria. Un proyecto de práctica pedagógica. I Encuentro Nacional y II Distrital de Experiencias en Enseñanza de la Biología y de la Educación Ambiental, Santafé de Bogotá, agosto de 1996.

. ARIETA de MOLINA, J., REYES, L. y BONILLA, P. El sentido de la Biología en la enseñanza de las ciencias naturales. I Congreso Internacional en Enseñanza de las Ciencias Naturales y Exactas, Panamá, agosto de 1994.

FEYERABEND, P. K. 1984. Contra el método. Esquema de una teoría anarquista del conocimiento. Orbis: Buenos Aires. 189 p.

FREUD. S. 1993. Los textos fundamentales del psicoanálisis. Altaza: Barcelona. Pp. 487492

FULLAT. O. 1992. Filosofías de la educación. Paideia. CEAC: Barcelona

GEERTZ, O. 1996. La interpretación de las culturas. Gedisa: Barcelona

GIL-PEREZ, D Tres paradigmas básicos en la enseñanza de las ciencias. En: Revista Enseñanza de las Ciencias, 1983. Vol.I. No. 1. Pp. 26-33.

KLAFKI, W. Los fundamentos de una didáctica critico-constructiva. En: Revista Educación (Madrid). No. 280, mayo-agosto, 1986. Pp. 37-79. 
KLEIBER, G. 1995. La semántica de los prototipos. Visor Libros: Madrid

MELICH, J.C. 1994. Del extraño al cómplice. La educación en la vida cotidiana. Anthropos: Barcelona.

MELICH, J.C. 1996. Antropología simbólica y acción educativa. Papeles de Pedagogía. Paidós: Barcelona.

MIRANDA, L., ESPITIA, N. Diagnóstico sobre algunas nociones acerca de las plantas en niños de transición del IPN. 1995. Trabajo de grado (UPN).

MOCKUS, A. y otros. 1994. Las fronteras de la escuela. Sociedad Colombiana de Pedagogía: Bogotá.

O'LOUGHLIN M. Rethinking science education: Beyond Piagetian constructivism toward a sociocultural model of teaching and learning. J. Res. Sc. Teach. Vol. 29, No. 8,1992. Pp. 791-820.

PARRA SANDOVAL, R 1992. La calidad de la educación. Universidad y Cultura Popular. Bogotá. P. 253.

PIAGET, J. 1933. La representación del mundo en el niño. Morata: Madrid. $7^{\mathrm{a}}$ ed.

RODRIGO, M.J; RODRIGUEZ, A. y MARRERO, J. 1995. Las teorías implícitas Una aproximación al conocimiento cotidiano. Visor: Madrid

SARTRE, J-P 1993. El ser y la nada: Ensayo de ontología fenomenológica. Altaya: Madrid.

SAVATER, F. 1991. Ética para Amador. Madrid. Ariel

SHARDAKOV, M.N. 1963. Desarrollo del pensamiento en el escolar. Grijalbo: México.

SEGURA, D. 1993. Constructivismo: ¿construir qué? Bogota P 33 Mimeo

SMITH, C.U.M. 1975. El problema de la vida. Ensayo sobre los orígenes del pensamiento biológico. Alianza Universidad. Madrid.

TONUCCI, F. 1974. La investigación como alternativa a la enseñanza. 2a. ed. En: Cuadernos de Educación. Número Extraordinario (43).

WUKETITS, F.M. The history of the concept of life: a game of mind. En: Revista Ludus Vitalis Vol III, No. 4. 1995. Pp. 39-49.

ZULETA, E. 1995. Educación y Democracia. Un campo de combate. Bogotá: Corporación Tercer Milenio. 